\title{
Effet amnésiant des hypnotiques médicamenteux. Revue de la littérature. Cas personnels
}

\section{Sedative-hypnotic drugs and amnesia. Review and cases}

Jean-Pierre GOULLÉ*(1), Jean-Pierre ANGER ${ }^{(2)}$

(1) Laboratoire de Pharmacocinétique et de Toxicologie Cliniques, Groupe Hospitalier BP 24 - 76083 LE HAVRE Cedex - Tél : 0232733223 - Fax : 0232733238

(2) Laboratoire de Toxicologie, UFR Pharmacie, 2, avenue du Pr Léon Bernard - 35043 RENNES Cedex Tél : 0223234867 - Fax : 0223234794

* Auteur à qui adresser la correspondance : Dr Jean-Pierre GOULLÉ, Laboratoire de Pharmacocinétique et de Toxicologie Cliniques, Groupe Hospitalier du Havre - BP 24 - 76083 LE HAVRE Tél : 0232733223 -Fax : 0232733238 -e-mail : jgoulle@ch-havre.fr

(Reçu le 9 octobre 2002 ; accepté le 23 octobre 2002)

\section{RÉSUMÉ}

A partir d'une revue de la littérature, les auteurs font la synthèse des travaux réalisés sur l'effet amnésiant des hypnotiques médicamenteux. Ils s'intéressent en particulier à deux benzodiazépines : le flunitrazépam (Rohypnol ${ }^{\circledR}$ ) et le triazolam (Halcion ${ }^{\circledR}$ ) et à deux molécules apparentées le zolpidem $\left(\right.$ Stilnox $\left.x^{(}\right)$et le zopiclone (Imovane ${ }^{\circledR}$ ). Après un rappel sur la mémoire, la biologie de la mémoire, les différents types de troubles observés dans les processus de mémorisation, les effets amnésiants de ces quatre médicaments sont détaillés. Pour les quatre spécialités, il s'agit d'une amnésie antérograde dose dépendante. Si cet effet est contemporain de l'action hypnotique, les récepteurs sont distincts. Une entité clinique particulière, importante à connaître en médecine légale, est l'amnésie automatisme ou automatisme hypercomplexe amnésique. Pour illustrer cette revue de la littérature des observations médicales et médico-légales personnelles sont rapportées.

\section{MOTS-CLÉS}

Médicaments, hypnotiques, amnésie.

\section{SUMMARY}

This review describes amnesia following sedative-hypnotic drugs use. Anterograde amnesia clearly does occur with many benzodiazepins. Four drugs were studied : two benzodiazepins : flunitrazepam (Rohypnol ${ }^{\circledR}$ ) and triazolam (Halcion $^{\circledR}$ ) and two that are structurally unrelated to the benzodiazepins, but shares some of the their properties: zolpidem (Stilnox ${ }^{\circledR}$ ) and zopiclone (Imovane ${ }^{\circledR}$ ). The amnesic effects of these four drugs were described, memory process, biology of memory, and memory process impairment evoked. Anterograde amnesia has been shown to be dose dependent with the four drugs. This effect is associated with the hypnotic one, but the the receptors are different. For forensic medicine, an important and particular type of amnesia has to be known : amnesia automatism or amnesic complex automatism. At last four personnal cases of amnesia are reported.

\section{KEY-WORDS}

Drugs, sedative hypnotics, amnesia. 


\section{Introduction}

Les hypnotiques présentent un certain nombre d'effets indésirables : sédation résiduelle, rebond d'insomnie, dépendance physique, tolérance, dépression respiratoire, dépression partielle du système nerveux central (interaction possible avec d'autres médicaments), enfin des effets délétères sur la mémoire (1). En effet un certain nombre de médicaments appartenant à la classe des hypnotiques présentent la particularité d'exercer une action négative sur les capacités de mémorisation d'un individu, ou effet amnésiant. Dans le cadre de la recherche d'une action hypnotique, il s'agit d'un effet secondaire indésirable. Mais parfois celui-ci est au contraire souhaité :

- pour améliorer la tolérance et l'acceptation des actes douloureux, chirurgicaux, ou invasifs de diagnostic par exemple (2),

- en cas de soumission chimique à des fins délictuelles ou criminelles (vol, viol, crime).

Dans ce contexte, les produits agissant vite -souvent dans les 20 minutes- sont particulièrement prisés : flunitrazépam (FNZ), triazolam (TRZ) (3). Outre une activité amnésiante, ils exercent divers effets également recherchés : désinhibition, passivité, perte de la volonté de résister, relaxation musculaire (3). Ces effets sont potentialisés par l'alcool. Les hypnotiques médicamenteux comprennent certaines benzodiazépines (BZD), deux spécialités apparentées : zolpidem (ZLP Stilnox $(\mathbb{B})$ et zopiclone (ZPC - Imovane $($ ), ainsi que des antihistaminiques. Les propriétés amnésiantes des BZD ont été découvertes rapidement après leur introduction en pratique anesthésique (4). En 1970 Clarke (5), dans une étude placebo contrôlée, montre que 10 min après injection intraveineuse de diazépam, les malades présentent une forte amnésie antérograde.

L'histoire des hypnotiques médicamenteux a connu trois révolutions importantes : la première (1970) avec l'introduction d'une $\mathrm{BZD}$, le flurazépam, alors que cette classe thérapeutique était jusqu'alors essentiellement constituée par des barbituriques ; la seconde (1980) avec l'apparition des BZD à demi vie plus courte : FNZ, TRZ ; la troisième (1990) avec la naissance de nouveaux hypnotiques n'appartenant pas aux BZD : ZLP et ZPC.

Des particularités doivent être signalées, ainsi le flurazépam est commercialisé dans certains pays européens mais pas en France. Le FNZ (Rohypnol ${ }^{\circledR}$ ) et son générique sont disponibles en France mais interdits aux Etats-Unis en raison du grand nombre de cas de soumission chimique constatés.

\section{Mémoire et amnésies}

La mémoire est conçue comme l'ensemble des mécanismes neurobiologiques et psychologiques qui permettent le stockage et la récupération d'informations simples ou complexes (figure 1). Elle nous permet de connaître, de nous repérer et d'évoluer dans le monde qui nous entoure. Dans le système mnésique, les informations traitées superficiellement se mémorisent bien moins que les informations traitées en profondeur et associées à des connaissances existantes. La mémoire ayant pour base biologique le cerveau, certains fonctionnements sont directement liés au bon fonctionnement du cerveau, d'autres sont purement une "programmation" psychologique ou enfin dus à la combinaison des effets biologiques et psychologiques.

Les conceptions modernes de la mémoire sont très influencées par le développement de l'informatique et la mémoire est le plus souvent conçue comme un ensemble de modules synthétisant et stockant des informations selon des modes variés, les codes. Entre la réception des signaux physiques par les organes des sens (œil, oreille...) et le rappel des souvenirs, il existe un grand nombre d'étapes de codage et d'information.

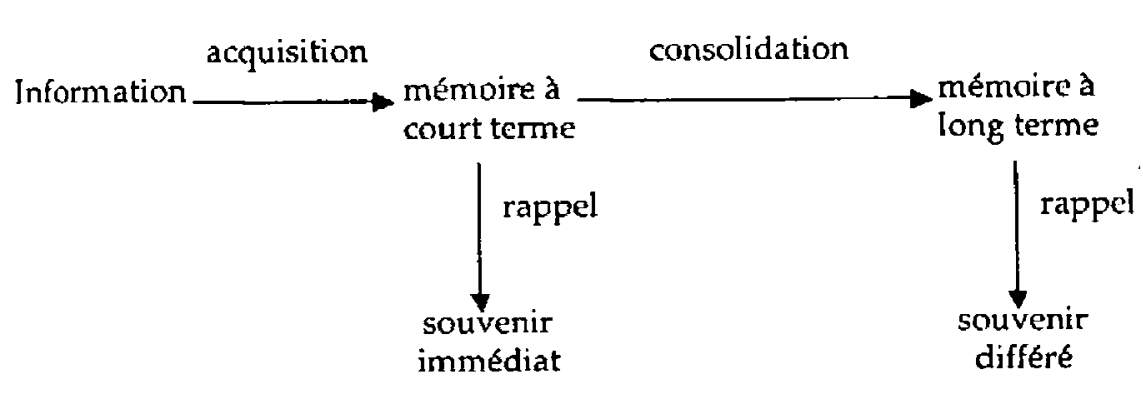

Figure I : Modèle du processus de mémorisation selon Cherkin et Harroun (51).

Classiquement on reconnaît trois grands types de mémoires :

1) la mémoire à court terme ou mémoire primaire : elle enregistre les informations afin d'assurer le raisonnement et la compréhension ;

2) la mémoire à moyen terme ou mémoire secondaire : elle permet le codage et le stockage durable des informations. On la subdivise en deux modules :

- la mémoire explicite : c'est elle qui conserve les souvenirs consciemment exprimés. Il s'agit à la fois de la mémoire épisodique ou autobiographique c'est à dire l'histoire personnelle du sujet mais aussi de la mémoire sémantique, fonction du savoir et de la culture du sujet.

- la mémoire implicite : elle est inconsciente ; elle nous permet de réaliser des taches automatiques (jouer 
au tennis, attacher un lacet...). Elle se subdivise également en mémoire procédurale ou mémoire motrice celle qui assure l'acquisition du savoir-faire, de la compétence du sujet et la mémoire émotive dont le centre névralgique est l'amygdale qui reçoit les informations en provenance du thalamus et du cortex.

3) la mémoire à très long terme ou mémoire tertiaire : elle concerne surtout des souvenirs de la mémoire sémantique et un peu moins de la mémoire épisodique. La mémoire chez l'homme implique l'intégrité anatomique et fonctionnelle de quelques structures ou zones du cerveau, bien identifiées. Il n'y a pas cependant de "centre de la mémoire" mais plutôt des circuits ou modules nécessaires au déroulement des différents stades de cette fonction cognitive.

Les troubles de la mémoire ont de multiples causes : ils peuvent être d'origine fonctionnelle (ex : la dépression anxieuse), liés à l'age (ex : l'oubli bénin de la sénescence), être induits par des médicaments ou enfin relever de perturbations anatomiques ou histologiques (démences).

Les amnésies ou perte de mémoire peuvent être classées en quatre groupes :

1) l'amnésie antérograde (AA) : c'est l'incapacité de mémoriser des faits nouveaux et donc d'apprendre ; on l'appelle aussi amnésie de fixation. Elle apparaît à la suite d'événements graves (arrêt cardiaque, asphyxie, traumatisme crânien). Elle peut également survenir après la prise de médicaments hypnotiques. Dans cette amnésie, la mémoire à court terme et la mémoire à long terme implicite (procédurale et émotionnelle) sont conservées, les souvenirs anciens ne sont pas affectés. On garde ses compétences mais pas le souvenir de les avoir acquises. Dans l'immense majorité des cas, les personnes atteintes d'AA ne se souviennent pas d'événements de leur routine (entretien avec quelqu' un, activité professionnelle, achat de nourriture) (6). Les sujets fonctionnement automatiquement en dépit de la réalisation de tâches complexes (conduite automobile, pratique de la médecine) (6). L'AA consécutive à la prise de médicaments hypnotiques est différente de celle observée après consommation d'alcool, avec ce dernier à l'AA s'ajoute une dégradation des performances. En ce qui concerne les médicaments hypnotiques, on constate que l'effet amnésiant augmente parallèlement à l'effet hypnotique, même si les mécanismes d'action sont différents (7). Au sein de ce tableau d'AA, il convient d'évoquer le syndrome «d'amnésie automatisme» qui associe : troubles du comportement, état confusionnel, attitude de consentement, suggestibilité, conduite automatique suivie d'amnésie antérograde totale ou quasi totale. Il peut être la conséquence de l'abus de certains médicaments hypnotiques (8).
2) l'amnésie rétrograde : c'est un déficit de mémorisation des souvenirs anciens. Ce type d'amnésie apparaît à la suite d'une lésion cérébrale. Les souvenirs antérieurs à celle-ci s'oublient peu à peu, les souvenirs les plus anciens étant les plus résistants à l'oubli. Cette amnésie est associée à des pathologies neuro-dégénératives comme la démence sénile ou la maladie d'Alzheimer.

3) le syndrome de Korsakoff : il se caractérise par des fabulations, des fausses reconnaissances, des pertes de mémoire. Ce syndrome est du à l'alcoolisme, à des tumeurs ou des traumatismes crâniens qui provoquent des lésions de certains centres nerveux.

4) les ictus mnésiques : ce sont des amnésies brèves (quelques heures), d'apparition brutale et précédées par une émotion forte. Les ictus sont causés par des traumatismes crâniens, l'alcoolémie ou alors résultent des effets secondaires consécutifs à la prise de certains médicaments (benzodiazépines, anticholinergiques). Ils peuvent enfin s'observer à la suite d'une prise involontaire d'une substance exogène, en cas de soumission chimique.

\section{Biologie de la mémoire}

Les progrès de la biologie permettent aujourd'hui d'entrevoir quelques uns des mécanismes qui, dans notre cerveau, commandent l'exercice da la mémoire. Les milliards de cellules nerveuses (neurones) qui constituent notre système nerveux communiquent entre elles par l'intermédiaire de substances chimiques (neuromédiateurs) sécrétées au niveau des synapses. Il y a des neuromédiateurs de type excitateur : acétylcholine (Ach), noradrénaline (NA), dopamine (DA) et d'autres, au contraire de type inhibiteur : acide gamma amino butyrique (GABA). On explique aujourd'hui l'effet sur le comportement de la plupart des psychotropes (hypnotiques, antidépresseurs, tranquillisants, convulsivants) par leur action sur ces différents neuromédiateurs.

Ainsi l'Ach est l'un des plus importants neuromédiateurs du cortex cérébral, l'étage supérieur de la pensée consciente. Des travaux récents de l'équipe de $R$. Jaffard (9) ont mis en évidence l'importance de certains systèmes neuronaux utilisant l'Ach dans la maturation des souvenirs. D'autre part certains troubles de la mémoire qui accompagnent la maladie d'Alzheimer ont pu être rattachés à des déficiences de certaines voies qui fonctionnent avec l'Ach. A coté le GABA occupe parmi les neuromédiateurs inhibiteurs une place essentielle puisque le fonctionnement de près d'un tiers des synapses est sous sa dépendance. Dès lors les molécules qui réduisent son activité sont convulsivantes et anxiogènes. A l'opposé, les molécules qui accroissent l'activité du GABA sont sédatives, anticonvulsivantes, anxiolytiques voire amnésiantes. Il en est ainsi des benzodia- 
zépines qui se lient à des récepteurs spécifiques étroitement reliés de façon fonctionnelle aux récepteurs $\mathrm{GABA}_{\mathrm{A}}$ dans le système nerveux central. Le GABA provoque l'ouverture de canaux chlore : la perméabilité au chlore de la membrane des neurones augmente, ce qui atténue l'action des stimulis dépolarisants. Les benzodiazépines n'agissent pas de façon directe mais augmentent l'affinité du GABA pour ses récepteurs si bien que pour une même concentration de GABA, on aura une liaison plus élevée au récepteur et un effet plus important. L'excitabilité de la cellule nerveuse est diminuée (figure 2).

L'effet amnésiant de l'éthanol résulte vraisemblablement du même mécanisme puisque ce composé augmente la transmission GABAergique dans certaines régions du cerveau. Cependant, on ne sait pas encore de façon précise s'il augmente l'affinité du GABA pour le récepteur, s'il joue sur un site co-agoniste ou si cela se passe à un niveau différent.

Si le mécanisme intime de l'amnésie liée à une consommation abusive d'éthanol reste encore à définir, de nombreux travaux cliniques se sont par contre efforcés d'expliquer les pertes de mémoire induites par diverses molécules hypnotiques : les benzodiazépines et substances apparentées.

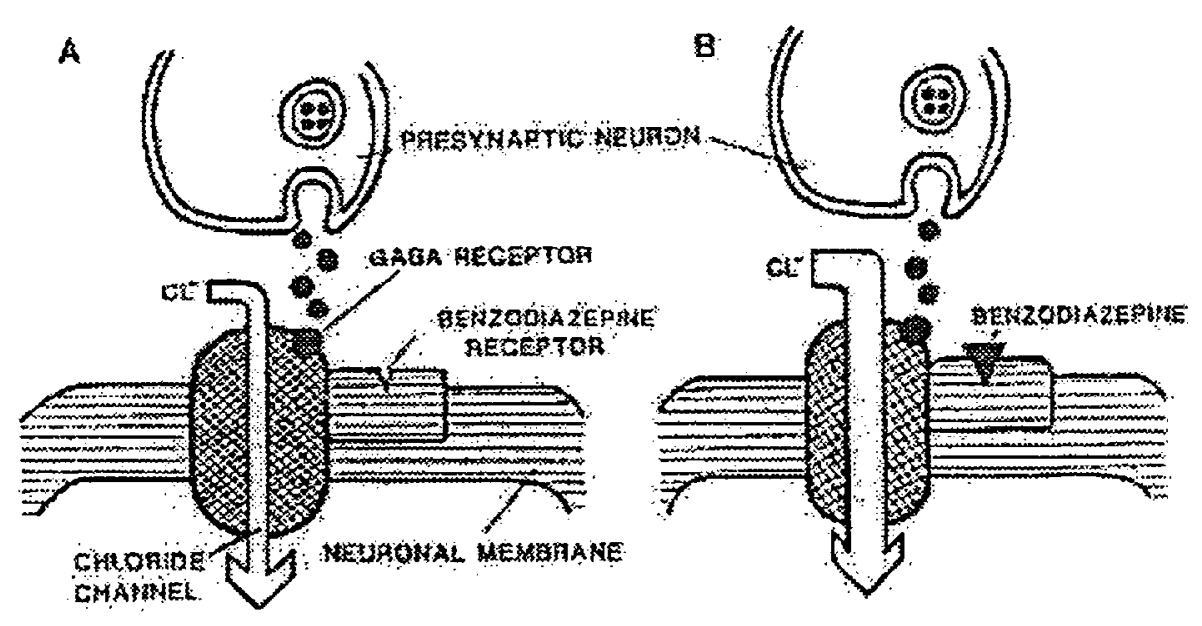

Figure 2 : Mode d'action des $B Z D$ sur les récepteurs $G A B A$ selon Loeffler (52):

- A : le GABA, en provoquant l'ouverture des canaux chlore, atténue l'action des stimulis dépolarisants,

- $B$ : la $B Z D$, en se fixant sur le récepteur GABA, augmente l'affinité du récepteur vis à vis du GABA, accroît le passage des ions chlorures et empêche ainsi la dépolarisation du neurone. L'excitabilité de la cellule nerveuse est diminuée.

\section{Les médicaments hypno- tiques}

Au dictionnaire Vidal électronique 2002 (10) figurent : - des antihistaminiques $\mathrm{H}_{1}$ : alimémazine (Théralène ${ }^{\circledR}$ ), clorazépate + phénothiazines $\left(\right.$ Noctran $\left.^{\circledR}\right)$, doxylamine $\left(\right.$ Donormyl ${ }^{\circledR}$, Lidene $^{\circledR}$, Noctyl $\left.{ }^{\circledR}\right)$, méprobamate + phé- nothiazine (Méprönizine ${ }^{\circledR}$ ), niaprazine $\left(\right.$ Nopron $\left.^{\circledR}\right)$, prométhazine (Phénergan ${ }^{\circledR}$ ),

- sept BZD : FNZ (Rohypnol ${ }^{\circledR}$ ), TRZ (Halcion ${ }^{\circledR}$ ), estazolam (Nuctalon ${ }^{\circledR}$ ), loprazolam $\left(\right.$ Havlane $\left.^{\oplus}\right)$, lormétazépam $\left(\right.$ Noctamide $\left.^{\circledR}\right)$, nitrazépam (Mogadon ${ }^{\circledR}$ ), témazépam (Normisson ${ }^{\circledast}$ ),

- deux médicaments apparentés : ZLP (Stilnox $\left.{ }^{\circledR}\right)$ et ZPC (Imovane ${ }^{\circledR}$ ).

A l'exception du Noctran ${ }^{\otimes}$ qui contient du clorazépate, les antihistaminiques $\mathrm{H} 1$ ne sont pas susceptibles d'induire d'amnésie. Les médicaments hypnotiques pouvant être à l'origine d'amnésies antérogrades sont les $\mathrm{BZD}$ indiqués précédemment et les deux spécialités apparentées : Stilno ${ }^{\circledR}$ et Imovane ${ }^{\circledR}$. Nous nous sommes intéressés aux deux BZD citées le plus souvent pour leur effet amnésiant : FNZ (Rohypnol ${ }^{\circledR}$ ), TRZ $\left(\right.$ Halcion $\left.^{\circledR}\right)$ et aux deux médicaments apparentés : ZLP $\left(\right.$ Stilnox $\left.^{\circledR}\right)$ et ZPC (Imovane ${ }^{\circledR}$ ).

\section{Benzodiazépines (FNZ, TRZ)}

Leur pharmacodynamie ainsi que leur pharmacocinétique sont bien documentées (10-12). Les caractéristiques essentielles sont reportées dans le Tableau I.

\section{Molécules apparentées (ZLP, ZPC)}

La synthèse des principales propriétés pharmacologiques et pharmacocinétiques peut être consultée dans le Tableau I (10,13-16).

\section{Les effets amnésiants FNZ (Rohypnol ${ }^{\circledR}$ ) et TRZ (Halcion ${ }^{\circledR}$ )}

L'induction d'une amnésie antérograde a d'abord été rapportée après administration de BZD utilisées en prémédication anesthésique ou lors d'examens endoscopiques $(5,17-19)$. Il a pu être montré que le déficit mnésique observé est particulièrement intense avec l'ensemble des $1,4 \mathrm{BZD}$, ce qui n'est pas le cas après administration de 1,5 BZD comme le clobazam par exemple $(20,21)$. L'hypothèse la plus couramment avancée pour expliquer les troubles aigus de la mémoire liés à l'emploi de BZD est un déficit de l'acquisition et de la fixation à long terme. Ce déficit serait alors en rapport avec un trouble de la vigilance induit par l'activité hypnotique de ces substances $(22,23)$. Cependant les effets amnésiques du FNZ sont indépendants du sommeil ; ils sont concomitants à l'induction des effets sédatifs mais indépendants de la sédation (24). L'emploi de deux antagonistes spécifiques a permis de montrer que les récepteurs cérébraux des effets sédatifs sont différents de ceux contrôlant les effets amnésiques (24). La fréquence de l'amnésie antérograde apparaît être en relation avec la dose (25-28), et la voie d'administration, mais pas obligatoirement avec la concentration plasma- 


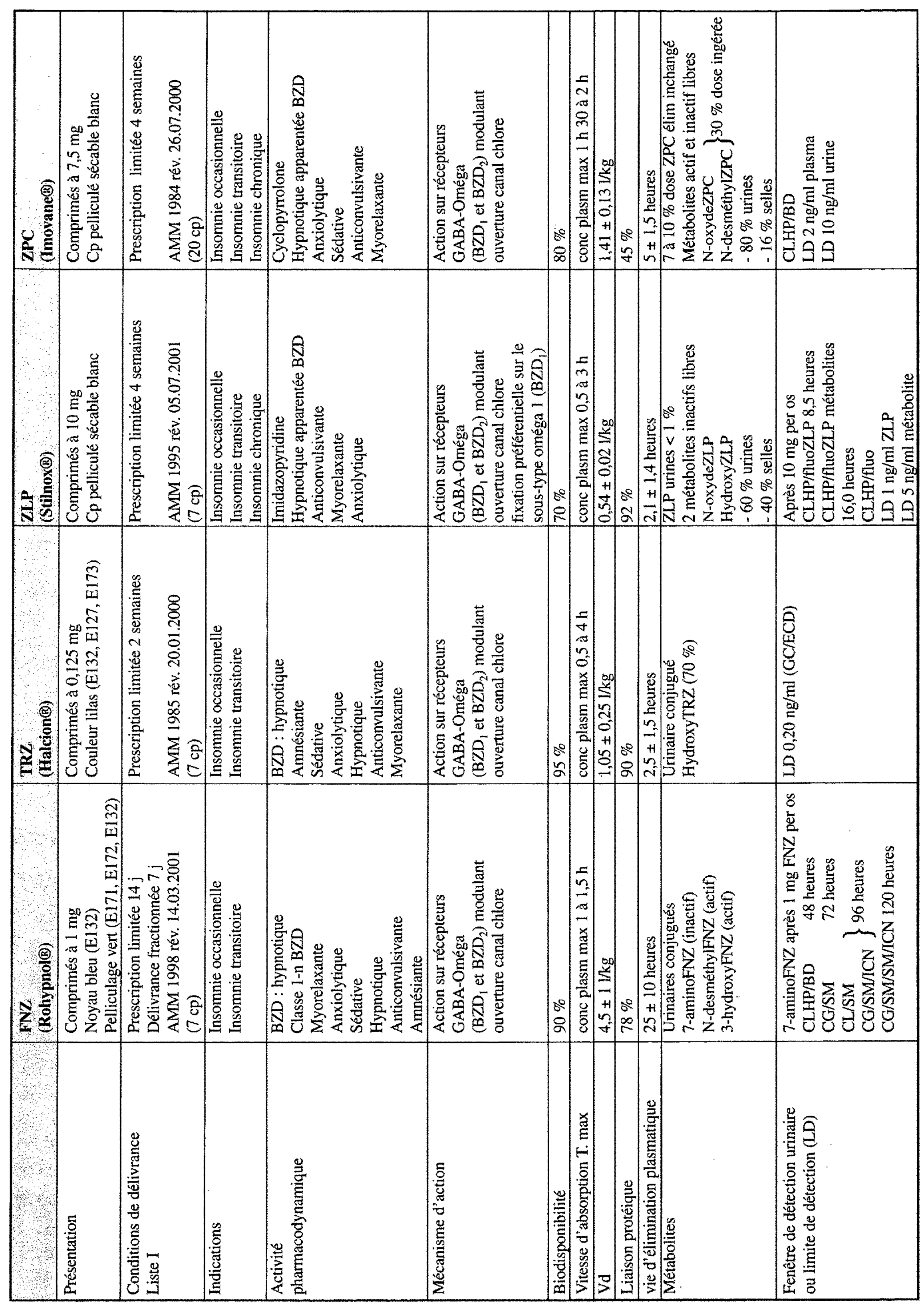


tique (29). Ainsi l'effet amnésique du diazépam est supérieur après administration IV par rapport à l'injection IM.

Pour Klein (30) les effets observés après TRZ $(0,5 \mathrm{mg})$ et après FNZ (1 mg) indiquent que l'amnésie antérograde induite par ces deux produits semble bien liée à un déficit des processus d'acquisition. En effet, les performances mnésiques sont particulièrement dégradées et de façon précoce par ces deux BZD au cours de l'apprentissage alors que d'autres BZD les affectent peu. Ces résultats laissent donc supposer que la dégradation du rendement mnésique, lors des évocations différées ne semble pas liée à l'incapacité des sujets à évoquer un matériel qui aurait été correctement appris, mais bien à l'absence de consolidation et de stockage de l'information présentée lors de l'apprentissage (30).

Selon Roth et al (22), l'absence de consolidation serait secondaire à une réduction de la latence d'endormissement après traitement par BZD. Cette hypothèse a été confirmée par Roehrs et al (25) dont les résultats indiquent que l'apprentissage et le stockage ne sont correctement réalisés que si la durée d'éveil est suffisante pour permettre la consolidation mnésique ; une vigilance normale et une période critique d'éveil de l'ordre de 5 minutes sembleraient nécessaires pour réaliser cette consolidation. Ainsi chez un sujet, il a pu être vérifié d'une manière ponctuelle que le maintien volontaire de la vigilance pendant les $3 \mathrm{~h}$ qui ont suivi la prise de $0,5 \mathrm{mg}$ de TRZ, permettait de conserver intactes les performances mnésiques lors des évocations à $8 \mathrm{~h}$ et à $24 \mathrm{~h}$ après la prise du produit (30). Ces résultats montrent par ailleurs que le déficit du rendement mnésique est précoce et intense. Il peut être mis en évidence dès la $30^{\grave{e}}$ min après absorption de TRZ et de FNZ chez les sujets ne présentant pas comportementalement des troubles apparents de la vigilance (30). La dégradation précoce et rapide de la performance mnésique après TRZ et FNZ pourrait être liée à des vitesses d'absorption et des concentrations tissulaires différentes avec les 1-5 BZD. Cet effet rapide se manifeste sous forme d'une perte de mémoire transitoire, véritable ictus mnésique présenté par un sujet une heure après absorption de TRZ : oubli de sa rencontre avec l'expérimentateur et oubli d'avoir été en situation d'apprentissage de test $30 \mathrm{~min}$ avant (30). Aucune corrélation par contre n'a pu être montrée jusqu'ici entre la concentration plasmatique et l'amnésie antérograde dont elles sont responsables $(29,31)$.

Il convient d'ajouter qu'outre l'AA, le FNZ présente la particularité d'entraîner des pertes d'inhibition (28,32-34).

Le FNZ en cas d'abus peut être à l'origine du tableau clinique particulier «d'amnésie automatisme» décrit plus haut encore appelé « automatisme hypercomplexe amnésique ». Il s'agit de la consommation abusive de
FNZ fréquemment rencontrée chez les toxicomanes aux opiacés. Salvaggio (8) chez une série de 53 toxicomanes constate une consommation moyenne de 20 $\mathrm{mg} / \mathrm{j}$ de FNZ -en une prise dans $50 \%$ des cas- pouvant aller jusqu'à $80 \mathrm{mg} / \mathrm{j}$ !. Le syndrome «d'amnésie automatisme» est très fréquent et peut aussi être à l'origine d'actes délictueux. Dans $11 \%$ des cas les sujets présentent une sensation d'invincibilité pouvant les pousser à des actes violents. Ce syndrome a été bien décrit par Rager en 1986 (35) sous le nom «d'automatisme hypercomplexe amnésique». Sa durée est de quelques heures, le sujet ne conserve aucun souvenir de cette période (accident d'automobile, acte bizarre, conduite agressive), il présente un comportement inhabituel (agressivité, quérulence, exaltation)-comportement qui n'est pas immédiatement repéré comme pathologique par l'entourage-, l'activité déployée est complexe, coordonnée et témoigne d'une certaine adaptation aux variations de l'environnement, une rupture nette avec la personnalité habituelle ainsi qu'une altération du champ de conscience sont constatées (levée des inhibitions, perte des références éthiques). L'alcool, la fatigue, le manque de sommeil et le stress semblent constituer des facteurs favorisants.

Pour le TRZ, la détérioration cognitive apparaît se limiter à la consolidation et au stockage de la phase d'acquisition de la mémoire et à tous les aspects de la mémorisation qui requièrent un rappel conscient actif. L'amnésie antérograde se révèle dose-dépendante avec le TRZ (25). A la dose de 0,5 mg de TRZ, l'amnésie dure plus longtemps qu'avec $7,5 \mathrm{mg}$ de ZPC (26) et est comparable à celle résultant de l'administration d'au moins $15 \mathrm{mg}$ de ZLP (36). Elle est concomitante de l'action sédative du TRZ (26). Dans une revue de la littérature consacrée à cette molécule, Rothschild (6) relève que l'AA varie très largement en fonction des critères et des modes d'évaluation de l'amnésie (de 0,4 à $19 \%$ ). Une période minimale de vigilance de $5 \mathrm{~min}$ est essentielle pour produire une consolidation de la mémorisation chez des sujets recevant $0,5 \mathrm{mg}$ de TRZ (37). De nombreuses études ont été réalisées sur la mémoire antérograde et l'amnésie provoquée par les médicaments mais paradoxalement peu de travaux ont été consacrés à la mémoire rétrograde. Récemment Fillmore (38) a mis en évidence une amélioration de la mémoire rétrograde par le TRZ. Il avait déjà été constaté avec les BZD une facilitation des processus de mémorisation rétrograde $(39,40)$. Certains auteurs suggèrent que cela est dû aux effets de l'AA $(41,42)$.

\section{ZLP (Stilnox ${ }^{\circledR}$ ) et ZPC (Imovane ${ }^{\circledR}$ )}

\section{a) le ZOLPIDEM}

Le ZLP est une imidazopyridine de structure différente de celle des BZD, structure communément retrouvée 
dans un grand nombre d'hypnotiques conventionnels. Il ne possède pas d'effets sur la mémoire rétrograde mais, on a pu observer quelques effets sur la mémoire antérograde notamment à des doses supérieures à $20 \mathrm{mg}$ par prise (43). Le ZLP se lie sélectivement aux récepteurs $\mathrm{BZ}$ et potentialise l'effet inhibiteur du GABA via le complexe GABA-BZD. Par ailleurs, le ZLP potentialise davantage que les BZD l'efficacité du GABA à ouvrir les canaux chlore (44). Cependant le ZLP a une affinité plus marquée pour un sous-type de récepteur $B Z D$, le $B Z D(\omega 1)$ et sur le plan pharmacologique, il possède une action hypnotique et sédative différente de celle des BZD conventionnelles. Langer et al.,(45) ont rapporté que les récepteurs $\omega 1$ et $\omega 2$ étaient distribués de manière identique dans le cerebellum, le cortex cérébral et la substance noire; par contre les récepteurs $\omega 2$ sont prédominants dans le noyau accumbens, l'hippocampe et le noyau caudate. Puisqu'il y a peu de récepteurs $\omega 1$ dans l'hippocampe, zone particulièrement impliquée dans la mémoire, on en a conclu que le ZLP exerçait des effets moindres sur la mémoire que les BZD hypnotiques traditionnelles qui ne se lient pas sélectivement à ces récepteurs et diverses études ont été menées pour prouver cette hypothèse. Balkin et al. (46) montrent ainsi chez 12 hommes adultes en bonne santé que $20 \mathrm{mg}$ de ZLP et $5 \mathrm{mg}$ de TRZ étaient équivalents au niveau de l'action hypnotique mais constatent une détérioration cognitive seulement dans le groupe TRZ. Ils confirment ainsi le rôle respectif des deux soustypes de récepteurs $\omega 1$ et $\omega 2$. Rohers et al (7), en 1994 ont observé que 10 et $20 \mathrm{mg}$ de ZLP et 0,25 et $0,5 \mathrm{mg}$ de TRZ affectent à la fois les fonctions psychomotrice et cognitive peu de temps après l'administration, mais ces effets ont disparu le matin suivant. Ils en concluent que le dysfonctionnement psychomoteur et la détérioration de la mémoire attribuables aux hypnotiques sont directement reliés à l'effet hypnotique des adjuvants du sommeil. Suzuki et al (47) testèrent les effets de $10 \mathrm{mg}$ ZLP, $0,25 \mathrm{mg}$ de TRZ et $5 \mathrm{mg}$ de nitrazépam (NZP) sur la fonction cognitive chez des hommes adultes en bonne santé. Le ZLP aussi bien que le TRZ ont produit une amnésie le matin suivant le test qui avait été présenté 2 heures après l'administration, avant d'aller dormir. Seuls les groupes NZP et TRZ présentèrent des effets résiduels le matin suivant. Le syndrome «d'amnésie automatisme» a également été décrit avec le ZLP (48).

\section{b) le ZOPICLONE}

Le ZPC est un hypnotique d'action courte avec une structure cyclopyrrolone qui exerce son action en se liant aux récepteurs $\mathrm{BZD}$ mais également aux récepteurs $\omega 1$ comme le ZLP. Warot et al. (1987) (49) ont rapporté que $7,5 \mathrm{mg}$ de $\mathrm{ZPC}$ et $0,25 \mathrm{mg}$ de TRZ indui- sent significativement une amnésie antérograde qui affecte la mémoire à court et à long terme mais les effets hypnotiques de ces deux molécules restent identiques. Isawa et al. (50) observent que $10 \mathrm{mg}$ de ZLP et 7,5 mg de ZPC n'entraînent pas d'amnésie rétrograde tant qu'ils sont utilisés à des posologies standard et bien que le ZLP possède plus d'affinité pour les récepteurs $\omega 1$ que le ZPC, il n'y a aucune différence entre les effets sur la fonction cognitive aux posologies standard utilisées.

\section{Observations médicales et médico-légales}

\section{Amnésie et vol}

Un collégien de 13 ans est admis aux urgences pédiatriques du CHU de Rouen en coma stade II. Un camarade lui aurait donné des bonbons de couleur bleue. L'analyse d'un extrait des urines par chromatographie en phase gazeuse couplée à la spectrométrie de masse (CG-SM) révèle la présence de 7-amino-FNZ, ainsi que de FNZ. L'interrogatoire du camarade qui lui avait donné les bonbons révèlera que ce dernier voulait lui voler sa calculatrice.

\section{Amnésie et vol}

Un homme de 55 ans consulte en neurologie pour un trouble de mémoire et de la motricité survenus lors d'un déplacement professionnel à Montpellier. Monsieur X... ne prend aucun médicament. Il dit avoir dîné avec des amis, s'être couché tôt et s'être réveillé le lendemain à 16 heures. A son lever, il tombe et titube durant plusieurs heures. Il regagne Rouen en train avec difficultés en passant par Bordeaux. Il garde de ce voyage peu de souvenirs, changeant de trains avec une démarche ébrieuse, somnolent et ralenti sur le plan intellectuel. Arrivé à son domicile, il dort près de 24 heures et ne se souvient pas avoir mangé ou bu durant cette période. Il constate alors que ses espèces, ainsi que des chèques ont disparu. Trois jours après les faits, inquiet de son état de santé, il consulte son médecin qui l'adresse en Neurologie ou sont prélevés du sang et des urines au $4^{\mathrm{ime}}$ jour pour analyse toxicologique. Elle révèle la présence de traces de benzodiazépine dans les urines lors du dépistage. Celle-ci est confirmée par chromatographie en phase liquide couplée à la spectrométrie de masse (CL-SM) : il s'agit de 7-acétamidoclonazepam. La CL-SM permet de mettre encore en évidence des traces de clonazépam (Rivotrilß) dans le sang prélevé le $4^{\mathrm{im}}$ jour et prouve que l'intéressé a été victime de soumission chimique. Il se rappellera ultérieurement avoir bu avec une relation de passage un Orangina amer. 


\section{Amnésie et viol}

Une jeune femme se présente un lundi après-midi à l'hôtel de police de Lisieux. Elle prétend avoir été victime d'un viol le samedi précédent mais ne se souvient plus de rien. Elle aurait consommé des boissons alcoolisées au cours de la soirée du samedi mais n'a aucun souvenir de la fin de sa soirée ni de la nuit qui a suivi. Des prélèvements de sang et d'urines sont réalisés en vue d'une expertise toxicologique. L'examen des urines par immunoanalyse montre l'absence de BZD. L'analyse d'un extrait des urines par CG-SM atteste de manière formelle la présence de bromazépam (Lexomil $\left.{ }^{\circledR}\right)$.

\section{Amnésie automatisme et crime}

Une toxicomane de 45 ans, a l'habitude de consommer de grosses quantités de Rohypnol ${ }^{\circledR}$ (dosé $2 \mathrm{mg}$ à l'époque des faits en 1996) : une plaquette de 10 comprimés en une prise et même parfois la boite entière (40 mg). Un jour, après avoir consommé une boite entière, elle s'endort. L'ami avec lequel elle vit, excédé par ses prises répétées la réveille et lui reproche cette consommation excessive. Elle tente alors par deux fois de se jeter par la fenêtre. Ensuite ils jouent tous les deux au cow-boy avec une carabine 22 long rifle. Elle s'empare de l'arme et tire dans la poitrine de son concubin à bout portant. La balle lui traverse le corps majs par miracle, il s'en sortira indemne. La femme entendue par les enquêteurs indiquera qu'elle ne conserve pas le moindre souvenir de cet évènement.

\section{Conclusion}

A l'occasion de cette revue de la littérature, il nous a paru important de rappeler que parmi les effets secondaires des médicaments hypnotiques, l'apparition d'une amnésie est possible. Cette amnésie de type antérograde peut être observée avec toutes les BZD et avec deux spécialités apparentées (Stilnox ${ }^{\circledR}$, Imovane ${ }^{\circledR}$ ), mais pas avec les hypnotiques antihistaminiques $\mathrm{H} 1$. La mise en évidence de ces substances dans les milieux biologiques ne peut donc se limiter à des recherches par immunoanalyse. Elle impose le recours minimal à la $\mathrm{CPG}-\mathrm{SM}$ et à la CL-BD, voire à la CL-SM et à des techniques de «mass tandem», en fonction du délai séparant la prise supposée des recueils biologiques.

\section{Références}

1. Mendelson W.B., Jain B. An assessment of short-acting hypnotics. Drug Safety. $1995 ; 13: 257-70$.

2. O'Boyle C.A. Benzodiazepine-induced amnesia and anaesthetic practice : a review. Psychopharmacol. Ser. $1988 ; 6: 146-65$.

3. Schwartz R.H., Milteer R., LeBeau M. Drug-facilitated sexual assault ('Date rape'). Southern Medical Journal. $2000 ; 93: 558-61$.

4. Brandt A.L., Oakes F.F. Preanaesthesia medication : double-blind study of a new drug, diazepam. Anaesth. Analg. $1965 ; 44: 125-29$.

5. Clarke P.R.F., Eccersley P.S., Frisby J.P., Thornton J.A. The amnesic effect of diazepam. Br. J. Anaesth. 1970 ; $42: 690-97$.

6. Rothschild A.J. Disinhibition, amnestic reactions, and other adverse reactions secondary to triazolam : a review of the literature. J. Clin. Psychiatry. $1992 ; 53: 69-79$.

7. Roehrs T., Merlotti L., Zorick F., Roth T. Sedative, memory, and performance effects of hypnotics. Psychopharmacology. $1994 ; 116: 130-34$.

8. Salvaggio J., Jacob C., Schmitt C., Orizet C. Ruel M., Lambert $\mathrm{H}$. Consommation abusive de flunitrazépam par les toxicomanes aux opiacés. Ann. Med. Interne. 2000 : 151, suppl A : pp. A6-A9.

9. Galey D., Destrade C., Jaffard R. Relationships between septo-hippocampal cholinergic and the improvement of long-term retention produced by medial septal electrical stimulation in two inbred strains of mice. Behav. Brain Res. 1994 ; 60 : 183-89.
10. www. vidalpro.net

11. Kintz P., Villain M., Cirimiele V., Goullé J.P., Ludes B. Usage criminel de substances psycho-actives : le problème de la durée de détection. Acta Clin. Belg. 2002-1; $57: 24-30$.

12. Perault M.C., Piriou A., Vandel B. Pharmacodynamie et pharmacocinétique des benzodiazépines. Toxicorama. $1996 ; 8(3): 5-10$.

13. Ascalone V., Flaminio L. Determination of zolpidem a new slepp-inducing agent, and its metabolites in biological fluids : pharmacokinetics drug metabolism and overdosing investigations in humans. J. Chromatogr. 1992 ; $581: 237-50$.

14. Holm K.J., Goa K.L. Zolpidem. An update of its pharmacology, therapeutic efficacy and tolerability in the treatment of insomnia. Drugs. $2000 ; 59: 865-89$.

15. Salva P., Costa J. Clinical pharmacokinetics and pharmacodynamics of zolpidem. Clin. Pharmacokinet. 1995 ; $29: 142-53$.

16.Edeki T., Robin D.W., Prakash C., Blair I.A., Wood A.J.J. Sensitive assay for triazolam in plasma following low oral dose. J. Chromatogr. 1992 ; 577 : 190-94.

17. Dundee J.W., George K.H. The amnesic action of diazepam, flunitrazepam and lorazepam in man. Acta Anaesthiol. Belg. $1976 ; 27: 3-11$.

18. Kortilla K., Linnoila M. Amnesic action of and skills related to driving after intravenous flunitrazepam. Acta Anaesth. Scand. $19676 ; 20: 160-8$. 
19. MacKay A.C., Dundee J.W. Effect of oral benzodiazepine on memory. Br. J. Anaesth. $1980 ; 52: 1247-57$.

20. Hindmarch I. Some aspects of the effects of clobazam on human psychomotor performance. Br. J. Clin. Pharmacol. $1979 ; 7: 77$ S-82S.

21. Nicholson A.N., Stone B.M. Activity of the hypnotics, flunitrazepam and triazolam in man. Br. J. Clin. Pharmacol. 1979; $7: 83$ S-88S.

22. Roth T., Hartse K.M., Saab P.G. The effects of flurazepam, lorazepam, and triazolam on sleep and memory. Psychopharmacol. (Berlin). 1981; $70: 231-7$.

23. Spinweber C.L., Johnson L.C. Effects of triazolam $(0,5$ $\mathrm{mg}$ ) on sleep, performance, memory, and arousal threshold. Psychopharmacol. (Berlin). $1982 ; 76: 5-12$.

24. Gentil V., Gorenstein C., Camargo C.H.P., Singer J.M. Effects of flunitrazepam on memory ant their reversal by two antagonists. J. Clin. Psychopharmacol. 1989 ; 9 ; 191-197.

25. Roehrs T., Zorick F., Sicklesteel J., Wittig R., Hartse K., Roth T. Effects of hypnotics on memory. J. Clin. Psychopharmacol. $1983 ; 3: 310-13$.

26. Hindmarch I., Sherwood N., Kerr J.S. Amnestic effects of triazolam and other hypnotics. Prog. NeuroPsychopharmacol. \& Biol. Psychiat. 1993 ; 17 : 407-413.

27. Smith K.M. Drugs used in acquaintance rape. J. Am. Pharm. Assoc. $1999 ; 39: 51-25$.

28. Smith K.M., Larive L.L., Romanelli F. Club drugs : methylenedioxymethamphetamine, flunitrazepam, ketamine hydrochloride, and $\gamma$-hydroxybutyrate. Am. J. Health-Syst. Pharm. $2002 ; 59: 1067-76$.

29. Bixler E.O., Scharf M.B., Soldatos C.R. et al. Effects of hypnotic drugs on memory. Life Sci. $1979 ; 25: 1379-88$.

30. Klein M.J., Patat A., Manuel C. Etude des effets sur la mémoire et les performances psychomotrices induits chez le sujet sain par 3 benzodiazépines hypnotiques (triazolam, flunitrazépam, lorazépam). Therapie. 1986 ; 41 : 299-304.

31. Crevoisier Ch., Ziegler W.H., Eckert M., Heizmann P. Relationship between plasma concentration and effect of midazolam after oral and intravenous administration. $\mathrm{Br}$. J. Clin. Pharmacol. $1983 ; 16: 51$ S-61S.

32. National Institute on Drug Abuse. Community drug alert bulletin. http 165.112.78.61/clubdruga.html.

33. National Institute on Drug Abuse. Community Epidemiologic Work Group. Epidemiologic trends in drug abuse : advance report. Bethesda, MD : National Institutes of Health ; 1995 June.

34. Sporer K.A. Benzodiazepines. In : Olson KA. Poisoning and drug overdose. 2nd ed. Stamford, CT : Appleton \& Lange ; 1994.

35. Rager P., Bênézech M. Lacunes mnésiques et automatismes hypercomplexes après prise orale de benzodiazépines : aspects cliniques et médico-légaux. Annales Médico-Psycho. $1986 ; 144: 102-9$.

36. Scharf M.B., Mayleben D.W., Kaffeman M. Et al. Dose response effects of zolpidem in normal geriatric subjects. J. Clin. Psychiatry. $1991 ; 52: 77-83$.

37. Paquet C., Gaillard J.M., Tissot R. Flunitrazépam et mémoire. Acta Psychiatr. Belg. 1978 ; 78 : 374-82.
38. Fillmore M.T., Kelly T.H., Rush C.R., Hays L. Retrograde facilitation of memory by triazolam : effects on automatic processes. Physchopharmacol. $2001 ; 158$ : 314-21.

39. Parker E.S., Weingartner H. Retrograde facilitation of human memory by drugs. In : Weingartner $\mathrm{H}_{\text {., }}$ Parker E.S. (eds) Memory consolidation : psychobiology of cognition. Lawrence Erlbaum, New Jersey. 1984 : 23151 .

40. Weingartner H., Sirocco K., Curran V., Wolkowitz O. Memory facilitation following the administration of the benzodiazepine triazolam. Exp. Clin. Psychopharmacol. $1995 ; 3: 298-303$.

41. Curran H.V. Benzodiazepines, memory, and mood. Psychomarmacol. $1991 ; 105: 1-8$.

42. Curran H.V. Psychopharmacological perspectives on memory. In : Tulving E, Craik FIM (eds). The oxford handbook of memory. Oxford University Press, New York. 2000:539-54.

43. Langtry H.D., Benfield P. Zolpidem : a review of its pharmacodynamic and pharmacokinetic properties and therapeutic potential. Drugs. $1990: 40: 291-313$.

44.Zivkovic B., Perrault G., Morel E., Sanger D.J. Comparative pharmacology of zolpidem and other hypnotics and sleep inducers. In : Sauvanet J.P., Langer S.Z., Morselli P.L. (eds) "Imidazopyridines in sleep disorders : A novel experimental and therapeutic approach". Raven Press, New York, 1988 : 97-109.

45. Langer S.Z., Arbilla S., Scatton B., Niddam R., Dubois A. Receptors involved in the mechanism of action of zolpidem. In : Sauvanet J.P., Langer S.Z., Morselli P.L. (eds) Imidazopyridines in sleep disorders. RavenPress, New York. $1988: 55-80$.

46. Balkin T.J., O'Donnel V.M., Wesensten N., McCann U., Belenky G. Comparison of the daytime sleep and performance effects of zolpidem and triazolam. Psychopharmacol. $1992 ; 107: 83-88$.

47. Suzuki M., Uchiumi M., Murasaki M. Effects of a single dose of zolpidem, a novel benzodiazepine Benzodiazepine_1 receptor-related hypnotic, on human memory ; a comparative double-blind study with triazolam and nitrazepam. Jpn. J. Neuropsychopharmacol. $1993 ; 15: 375-89$.

48. Canaday B.R. Amnesia possibly associated with zolpidem administration. Pharmacotherapy. $1996 ; 16: 687$ 89.

49. Warot D., Bensimon G., Danjou Ph., Puech A.J. Comparative effects of zopiclone, triazolam and placebo on memory and psychomotor performance in healthy volunteers. Fundam. Clin. Pharmacol. $1987 ; 1$ : 145-52.

50. Isawa S., Suzuki M., Uchiumi M., Murasaki M. The effect of zolpidem and zopiclone on memory. Jpn. J. Neuropsychopharmacol. 2000; $20: 61-69$.

51. Cherkin A., Harroun P. Anaesthesia and memory processes. Anaesthesiology. 1971 ; 34 : 469-74.

52. Loeffler P.M. Oral benzodiazpines and conscious sedation. J. Oral Maxillofac. Surg. $1992 ; 50: 989-97$. 